\title{
Anhang IV: Einladung zum Diskussionsforum
}

KREBSGESELLSCHAFT E.V.

ERNSTVoN

BERGMANN

KLINIKUM

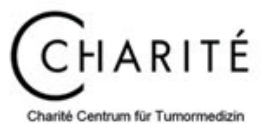

\section{Einladung zum Diskussionsforum: Sterben im Krankenhaus}

Ärztliche Erfahrungen mit der Behandlung schwerstkranker und sterbender Menschen im Krankenhaus - Eine qualitative Untersuchung.

Dienstag 21.01.2014

von 17:00 bis 19:30 Uhr

Charité Campus Virchow-Klinikum, Augustenburger Platz 1, 13353 Berlin

Forum 3, Lehrgebäude, Kursraum 1 im $2.0 G$

\section{Liebe Stationsärztin, lieber Stationsarzt,}

das Krankenhaus ist mit ca. 50 \% Sterbeort Nummer Eins in Deutschland - Tendenz steigend. Damit ist die Behandlung Schwerstkranker und Sterbender neben der Akutversorgung und Heilung eine zentrale Anforderung an Ärzte aller Fachdisziplinen im Krankenhaus.

Welche Erfahrungen haben Sie als Stationsarzt / Stationsärztin mit der Versorgung schwerstkranker und sterbender Menschen? Welche Ressourcen und Hilfen finden sich dafür an Ihrem Arbeitsplatz im Krankenhaus der Maximalversorgung - und welche fehlen? Was sind dabei die Herausforderungen vor dem Hintergrund der aktuellen gesellschaftlichen Debatten um ein würdevolles Sterben, der Entwicklungen der modernen Medizin sowie der zunehmenden Ökonomisierung im Gesundheitswesen?

Diese Fragen wurden im Rahmen der INSIDE-Studie (einer Kooperation der Charité Berlin und des Ernst-von-Bergmann Klinikums Potsdam, gefördert durch die Berliner Krebsgesellschaft) in Experteninterviews an Ärzte und Ärztinnen verschiedener medizinischer Fachdisziplinen gestellt. Denn es sind die Erfahrungsberichte aus dem Alltag der Stationsarbeit, die wichtige Hinweise für die Entwicklung von Strukturen und Empfehlungen liefern.

Nun wollen wir Ihnen die bisherigen Ergebnisse der Studie vorstellen, um diese mit Ihnen zu diskutieren und im Anschluss gemeinsam Empfehlungen abzuleiten. Dazu laden wir Sie herzlich zum Diskussionsforum am 21.01.2014 im Lehrgebäude, Forum 3 auf dem Campus Virchow-Klinikum der Charité ein. Wir freuen uns auf eine interessante Diskussion!

Die INSIDE-Studiengruppe

Asita Behzadi, Dr. Anja Hermann, Gesine Leithäuser und Dr. Peter Thuss-Patience

Verbindliche Rückmeldung bitte bis zum 14.01.2014 per:

E-Mail: asita.behzadi@charite.de oder Fax: 450553974

Rückfragen an Asita Behzadi unter Tel. 0175-4136285

Hiermit melde ich mich zum Diskussionsforum Sterben im Krankenhaus am 21.01.2014 an.

Name:

Medizinische Fachdisziplin: 
